\title{
A microwave and microfluidic planar resonator for efficient and accurate complex permittivity characterization of aqueous solutions
}

\author{
Thomas Chretiennot, Student Member, IEEE, David Dubuc, Member, IEEE, Katia Grenier, Member, \\ IEEE
}

\begin{abstract}
A microwave resonator is presented as a microfabricated sensor dedicated to liquid characterization with perspectives for chemistry and biology. The nanolitter range aqueous solution under investigation is located on top of the planar resonator thanks to a microfluidic channel compatible with a future lab-on-a-chip integration. The interaction between the electric field and the liquid translates into a predictable relationship between electrical characteristics of the resonator (resonant frequency and associated insertion loss) and the complex permittivity of the fluid (real and imaginary parts). A prototype of the resonator has been fabricated and evaluated with de-ionized water/ethanol mixtures, with ethanol volume fraction ranging from 0 to $20 \%$. Good agreement has been reached between theoretical and measured electrical parameters of the resonator. The discrepancy on the resonant frequency is estimated to $0.5 \%$, whereas the one on the associated transmission coefficient is lower than $1 \%$. This translates into a maximum relative error on the real and imaginary part of the predicted relative permittivity of less than $6.5 \%$ and $4 \%$ respectively, validating the principle of this accurate permittivity characterization methodology.
\end{abstract}

Index Terms-Permittivity measurement, microfluidics, microwave sensors, planar resonators.

\section{INTRODUCTION}

$\mathrm{R}$ $\mathrm{F}$ and microwave sensors are of major interest in biomedical applications [1], [2] as they offer numerous advantages compared to traditional techniques. These biosensors indeed do not require any markers, which avoids mandatory time-consuming preparatory steps of fluorescence's techniques [3], [4]. Moreover, such a method is fully compatible with a lab-on-a-chip approach: the sensor can be miniaturized, as already pointed out by the authors in [5].

In [5], we developed a miniature sensor for microwavebased broadband permittivity spectroscopy, which brings a rich set of information about liquid properties in a whole frequency range [6]. Nevertheless, such a characterization

This work was supported by the Centre National de la Recherche Scientifique (CNRS).

T. Chretiennot, D. Dubuc and K. Grenier are with :

CNRS, LAAS, 7 avenue du colonel Roche, F-31400 Toulouse, France, and

Univ de Toulouse, UPS, LAAS, F-31400 Toulouse, France. methodology is not straightforward and may require heavy and time-consuming data treatment [7], [8]. The development of efficient permittivity characterization is thus often based on resonant devices (whether it be planar resonators, dielectric resonators or metallic cavities) characterized by parameters such as their resonant frequency, peak attenuation or quality factor. These parameters are directly measurable from Sparameters (no elaborated computerized routine is needed). Moreover, the introduction of a chemical or biological medium in a specific area of the resonator modifies the electrical response, altering as well the resonant frequency and associated attenuation [9]. Thus, by simply monitoring those parameters, an efficient complex permittivity characterization method can be worked out.

The characterizations of a large range of chemical and biological liquids using dielectric or cylindrical resonators have already been successfully performed [10]-[14]. Appropriate sensitivity level and accuracy of such a technique was also demonstrated for lossy physiological medium in [14]. These references also point out the efficiency to consider resonant frequency and reflection/transmission coefficient at the resonant frequency (in spite of quality factor) in order to characterize both real and imaginary parts of the liquids' permittivity [10]- [14]. However, their typical size remains cumbersome and not suited to a lab-on-a-chip approach.

The device presented in this paper has been designed with the intention of gathering the advantages of (1) planar technologies for miniaturization [15]-[17], (2) RF resonators for characterization efficiency and accuracy. We have developed a compact sensor, suitable to a lab-on-a-chip approach and capable to perform an accurate and efficient characterization of the complex permittivity of fluids. The next two sections describe the sensor and its design and fabrication. Afterwards, a section is dedicated to the modeling of resonator's electrical characteristics shifts (resonant frequency and peak attenuation) due to complex permittivity variations. Finally, the last section gathers the experimental results together with model-based predicted data. 


\section{SENSOR DESCRIPTION AND OPERATION PRINCIPLe}

The sensor architecture consists of a planar folded quarterwavelength-type resonator etched in the central conductor of a coplanar waveguide. A microfluidic channel is bonded on the top face of the structure, perpendicularly to the central conductor. It conveys the liquid to be measured towards the sensing area. Fig. 1 depicts a schematic view of the device.

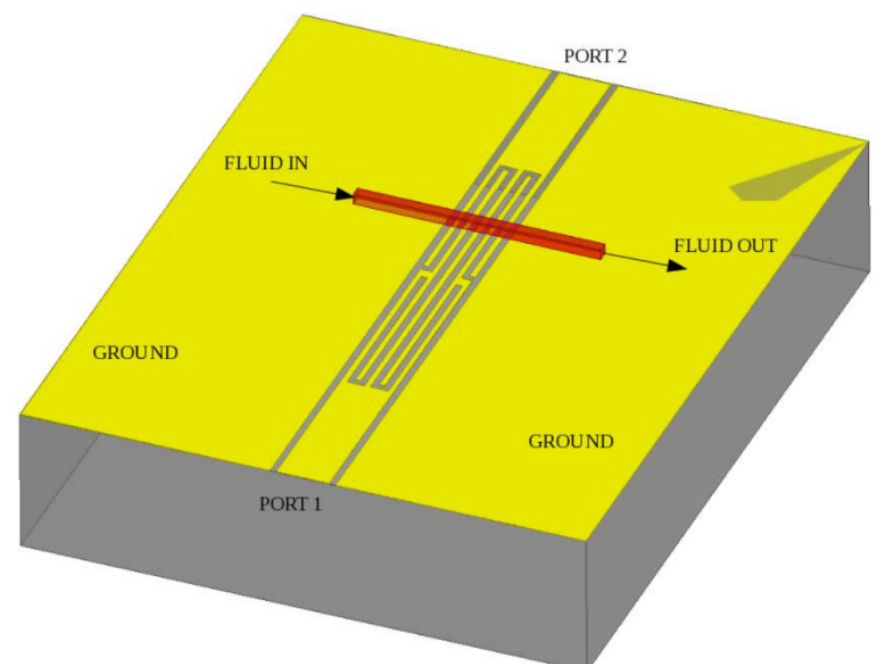

Fig. 1. Schematic view of the RF coplanar resonator with a microfluidic channel on top.

The principle of such a sensor is based on the interactions between the electromagnetic field and the fluid under test. When unfilled (e.g. when no fluid is introduced in the channel), the stop-band resonator is characterized by parameters such as resonant frequency, associated transmission loss and quality factor. When a fluid characterized by its complex permittivity is inserted in the microfluidic channel, the above listed parameters are modified and thus contain information relative to the fluid under test. The characterization of the complex permittivity is based on the quantification of the resonant frequency and peak attenuation shifts due to the fluid filling. Thereby, the abovedescribed device fulfills the three requirements:

- First, the compactness of the resonator is satisfied since the whole device is $1.03 \mathrm{~mm}$ wide (both grounds included) and $2.4 \mathrm{~mm}$ long,

- Then, the microfluidic channel enables measurements on nanolitter range liquids and is compatible with lab-on-a-chip applications,

- Eventually, the resonant-based measurement principle implies a quick and efficient sensing technique for the measurement of parameters such as the resonant frequency and associated loss.

\section{DESIGn AND FABRICATION}

The resonator architecture is inspired from those presented in [18]. Sizes of the resonator are optimized to match a 50ohm impedance standard and to reach the desired resonant frequency $(20 \mathrm{GHz})$. Central conductor is $0.18 \mathrm{~mm}$ wide and $2.4 \mathrm{~mm}$ long. Coplanar slots are $22 \mu \mathrm{m}$ wide. Both coplanar accesses are $0.55 \mathrm{~mm}$ long. As a result, the length of the resonant structure is $1.3 \mathrm{~mm}$. Gaps and metallic strips in the resonant structure are all $20 \mu \mathrm{m}$ wide. Metal layers are made of $0.3 \mu \mathrm{m}$-thick titanium/gold layers. Fig. 2 depicts the main sizes of the resonator.

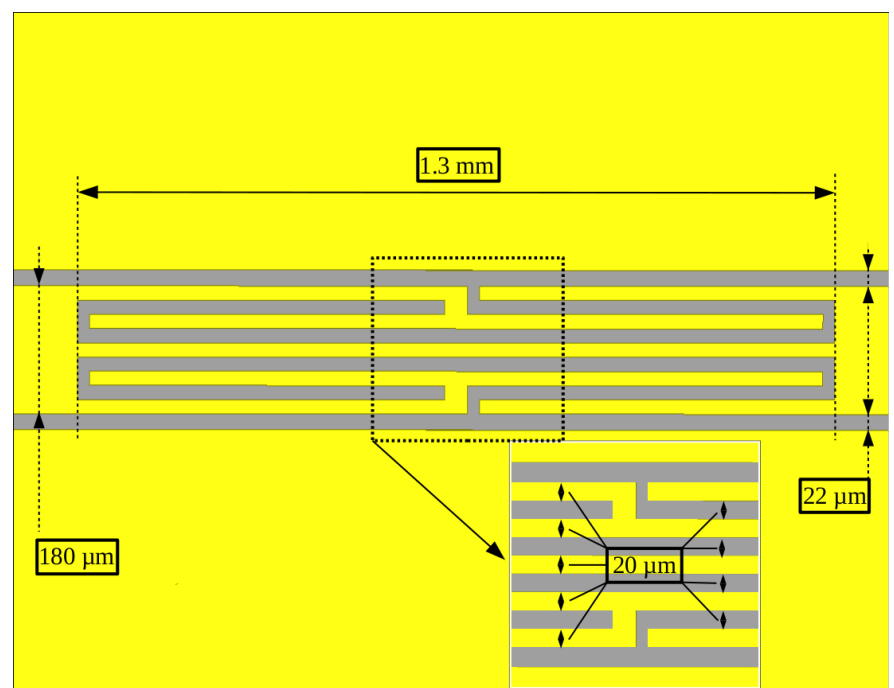

Fig. 2. Main sizes of the coplanar resonator.

The microfluidic channel is made of PolyDiMethylSiloxane (PDMS). The slice of the channel that crosses the resonant structure is $40 \mu \mathrm{m}$ wide and $40 \mu \mathrm{m}$ high. PDMS walls are 200 $\mu \mathrm{m}$ wide. The total height of the microfluidic structure (height of the channel plus height of the PDMS top wall) is approximately $1 \mathrm{~mm}$. The fabrication process of the presented device is divided into two main parts:

First, the microwave resonator is fabricated on a $0.5 \mathrm{~mm}$ thick quartz wafer. After its cleaning, a photoresist is spin coated on the top face and patterned by photolithography. Afterward, a $0.3 \mu \mathrm{m}$-thick Ti/Au layer is evaporated. The final step consists of a solvent wet etching in order to remove the remaining photoresist and the non-desired metal layers. The microfluidic channels are fabricated according to the following process: a silicon mold is fabricated by patterning a photoresist mask on a silicon substrate and then by applying a deep reactive ion etching step. Trenches in the silicon wafer correspond to the PDMS walls. Once the photoresist mask is removed, a PDMS layer is deposited on the mold. After curing at $75^{\circ} \mathrm{C}$, the PDMS channels are peeled off. Finally, the microfluidic channel is assembled on the microwave resonator. Further details about the fabrication process are available in [5]. 


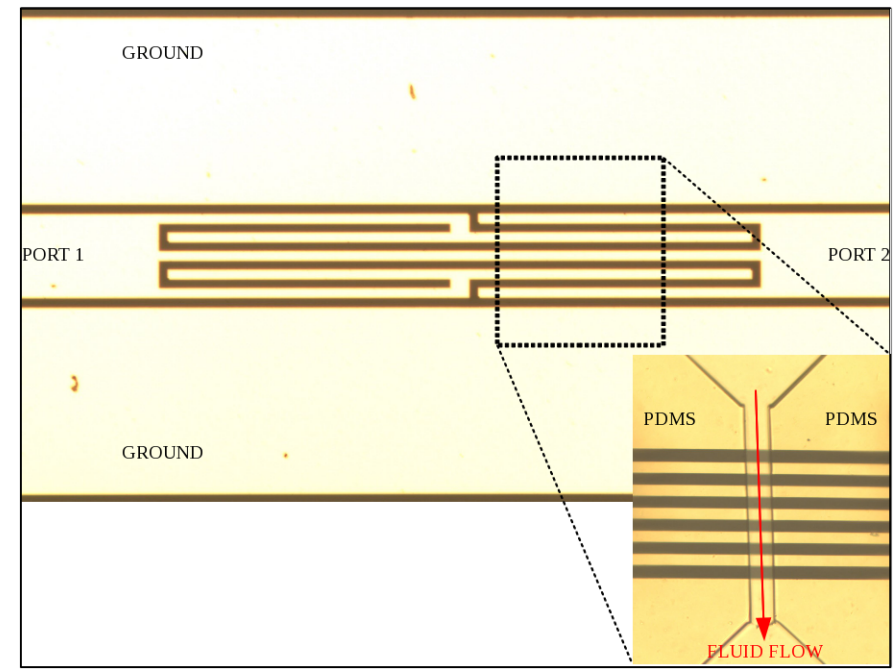

Fig. 3. Main view: fabricated prototype without microfluidic structure. Insert at the bottom right: zoom of the device in the area equipped with a microfluidic channel.

Fig. 3 shows a microphotograph of the fabricated device without any microfluidic structure. The insert at the bottom right of the picture shows a zoom in the central area of the resonator equipped with a $40 \mu \mathrm{m}$ per $40 \mu \mathrm{m}$ microfluidic channel.

The following paragraph describes the modeling of the electrical characteristics shifts (due to permittivity variations), which are obtained from the electromagnetic simulations of the above-described sensor.

\section{PREDICTIVE MODELING}

\section{A. Electromagnetic simulations}

The influence of the real ( $\left.\varepsilon^{\prime}\right)$ and imaginary ( $\left.\varepsilon^{\prime \prime}\right)$ parts of the liquid relative permittivity on resonant frequency and peak attenuation is first determined with the help of the 3D fullwave field solver High Frequency Structure Simulator (HFSS) from Ansoft [19]. The fluid relative complex permittivity $\varepsilon '-j$ $\varepsilon$ " is set to be $40-\mathrm{j}^{*} 40$, as it is the standard value of the complex permittivity of deionized water (DI water) around 20 $\mathrm{GHz}$ [20]. Then, $\varepsilon$ ' and $\varepsilon$ " are varied down to 20 and 24 respectively ( $\varepsilon$ ' takes 5 values: $40,36,32,24$ and $20 ; \varepsilon$ " takes 4 values : 40, 36, 32 and 24). In order to distinguish the impact of $\varepsilon$ ' from one side and the impact of $\varepsilon$ " from the other side, simulations have been performed as follows.

The real part $\varepsilon$ ' of the fluid complex permittivity varies from 20 to 40 while the imaginary part $\varepsilon$ " is maintained constant at 40. Such an operation is repeated for the other 3 values of $\varepsilon "(36,32,24)$. As a result, 20 values of the resonant frequency and peak attenuation corresponding to 20 couples of values for $\varepsilon$ ' and $\varepsilon$ '" are obtained. Such a simulation method enables to distinguish the influence of $\varepsilon^{\prime}$ ( $\varepsilon$ ' resp.) from the influence of $\varepsilon "$ ' $(\varepsilon$ resp.) on the resonant frequency (maximum attenuation resp.). Fig. 4.a and $b$ depict the resonant frequency and peak attenuation as a function of $\varepsilon$ ' and $\varepsilon "$ respectively. It clearly shows that the resonant frequency is more sensitive to variations of $\varepsilon$ ' than variations of $\varepsilon$ ', whereas the maximum notch attenuation is mainly driven by $\varepsilon$ ". By varying the real part $\varepsilon$ ' between 24 and 40 , the resonant frequency shifts are indeed about $230 \mathrm{MHz}$ (with the imaginary part $\varepsilon$ " maintained constant), whereas the resonant frequency shifts are less than $20 \mathrm{MHz}$ (with the real part $\varepsilon$ ' maintained constant), when the imaginary part $\varepsilon$ " is varied between 24 and 40 . The same situation arises for the maximum of insertion loss: its shift reaches $1.5 \mathrm{~dB}$, when $\varepsilon$ " is varied between 24 and 40 , whereas a variation of $\varepsilon$ ' in the same range only translates into a $0.06 \mathrm{~dB}$ contrast.

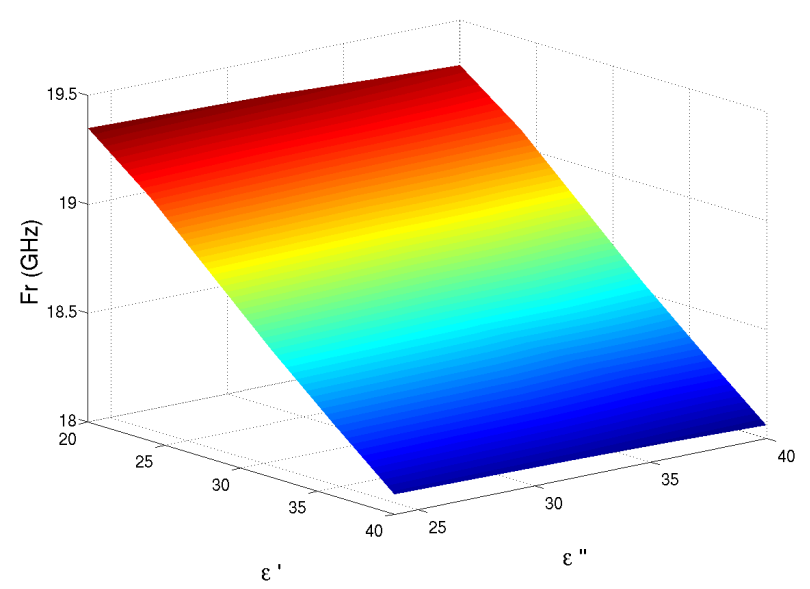

(a)

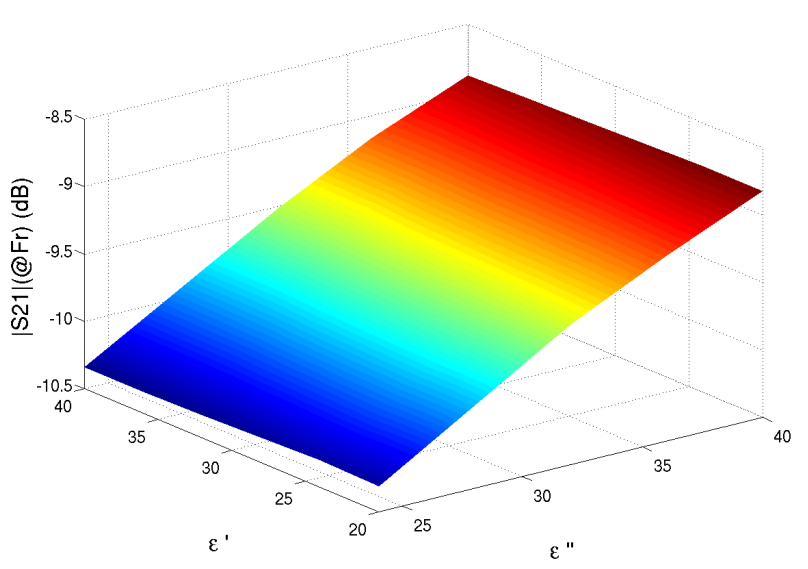

(b)

Fig. 4. 3D view of (a) the resonant frequency dependence and (b) the peak attenuation dependences on real and imaginary parts of the fluid complex permittivity.

\section{B. Predictive model derived from simulations}

Based on the simulations, a simple model for both resonant frequency and peak attenuation shifts due to complex permittivity variations is defined with the respective following equations:

$$
\begin{gathered}
\Delta F_{r}=\frac{\Delta F_{r}}{\Delta \varepsilon^{\prime}} \Delta \varepsilon^{\prime}+\frac{\Delta F_{r}}{\Delta \varepsilon^{\prime \prime}} \Delta \varepsilon^{\prime \prime}, \\
\Delta S 21=\frac{\Delta|S 21|}{\Delta \varepsilon^{\prime}} \Delta \varepsilon^{\prime}+\frac{\Delta|S 21|}{\Delta \varepsilon \varepsilon^{\prime \prime}} \Delta \varepsilon^{\prime \prime} .
\end{gathered}
$$

Such a model leads to the definition of four sensitive coefficients: 


$$
\begin{aligned}
& K_{1}=\frac{\Delta F_{r}}{\Delta \varepsilon^{\prime}} ; K_{3}=\frac{\Delta|S 21|}{\Delta \varepsilon^{\prime}} ;\left(\text { with } \varepsilon^{\prime \prime} \text { unchanged }\right) \\
& K_{2}=\frac{\Delta F_{r}}{\Delta \varepsilon^{\prime \prime}} ; K_{4}=\frac{\Delta|S 21|}{\Delta \varepsilon^{\prime \prime}} ;\left(\text { with } \varepsilon^{\prime} \text { unchanged }\right)
\end{aligned}
$$

where $\mathrm{K}_{1}\left(\mathrm{~K}_{2}\right.$ resp.) quantifies the impact of liquid's $\varepsilon$ ' ( $\varepsilon$ "' resp.) variations on the resonant frequency with $\varepsilon^{\prime \prime}\left(\varepsilon^{\prime}\right.$ resp.) maintained unchanged. Analogously, $\mathrm{K}_{3}\left(\mathrm{~K}_{4}\right.$ resp.) quantifies the impact of liquid's $\varepsilon$ ' ( $\varepsilon$ " resp.) variations on the peak attenuation with $\varepsilon$ " ( $\varepsilon$ ' resp.) maintained unchanged. With the chosen values for $\varepsilon$ ' and $\varepsilon$ ", four values of sensitivity coefficients $\mathrm{K}_{1}$ and $\mathrm{K}_{3}$ and five values of sensitivity coefficients $\mathrm{K}_{2}$ and $\mathrm{K}_{4}$ can be obtained. They are all reported in Tables I.a and I.b together with the respective mean values: $<\mathrm{K}_{1}>$ to $<\mathrm{K}_{4}>$ and standard deviations: $\sigma_{1}$ to $\sigma_{4}$.

TABLE I.a

SENSITIVITY COEFFICIENTS $\mathrm{K}_{1}$ AND $\mathrm{K}_{3}$ OBTAINED FROM SIMULATIONS

\begin{tabular}{ccc}
\hline \hline$\varepsilon^{\prime \prime}$ & $\mathrm{K}_{1}(\mathrm{MHz})$ & $\mathrm{K}_{3}$ \\
\hline 24 & -63.8 & $-0.07 \times 10^{-3}$ \\
32 & -63.1 & $-0.12 \times 10^{-3}$ \\
36 & -63.1 & $-0.08 \times 10^{-3}$ \\
40 & -62.5 & $-0.13 \times 10^{-3}$ \\
\hline & $<\mathrm{K}_{1}>=-63.1 \mathrm{MHz}$ & $<\mathrm{K}_{3}>=-0.10 \times 10^{-3}$ \\
& $\sigma_{1}=0.53 \mathrm{MHz}$ & $\sigma_{3}=0.028 \times 10^{-3}$ \\
\hline \hline
\end{tabular}

TABLE I.b

SENSITIVITY COEFFICIENTS $\mathrm{K}_{2}$ AND $\mathrm{K}_{4}$ OBTAINED FROM

\begin{tabular}{|c|c|c|}
\hline$\varepsilon^{\prime}$ & $\mathrm{K}_{2}(\mathrm{MHz})$ & $\mathrm{K}_{4}$ \\
\hline 20 & -3.7 & $3.6 \times 10^{-3}$ \\
\hline 24 & -1.9 & $3.6 \times 10^{-3}$ \\
\hline 32 & -2.1 & $3.6 \times 10^{-3}$ \\
\hline 36 & -2.3 & $3.5 \times 10^{-3}$ \\
\hline \multirow[t]{3}{*}{40} & -2.4 & $3.5 \times 10^{-3}$ \\
\hline & $<\mathrm{K}_{2}>=-2.5 \mathrm{MHz}$ & $<\mathrm{K}_{4}>=3.56 \times 10^{-3}$ \\
\hline & $\sigma_{2}=0.7 \mathrm{MHz}$ & $\sigma_{4}=0.055 \times 10^{-3}$ \\
\hline
\end{tabular}
SIMULATIONS

Both series are characterized by a weak standard deviation $\sigma$. Consequently, the model for resonant frequency shifts can be defined in terms of mean values for the sensitivity coefficients. Thus, it comes:

$$
\begin{gathered}
\Delta F r=K_{1} \Delta \varepsilon^{\prime}+K_{2} \Delta \varepsilon^{\prime \prime}=-63.1 \Delta \varepsilon^{\prime}-2.5 \Delta \varepsilon^{\prime \prime}(M H z) \\
\Delta|S 21|=-0.10 \times 10^{-3} \Delta \varepsilon^{\prime}+3.56 \times 10^{-3} \Delta \varepsilon^{\prime \prime} .
\end{gathered}
$$

Equations (5) and (6) linearly link the complex permittivity contrast between two liquids with the measured shift of the resonator's electrical characteristics. It is consequently possible to deduce liquid's properties from S-parameters measurements in a very simple and efficient manner and assuming:

(1) the use of liquids featuring both real and imaginary part of the permittivity in the range of $[20 ; 40]$,

(2) a known reference liquid from which contrasts will be evaluated.

The next paragraph proposes a simplification of the previously presented model, for DI water/ethanol mixtures investigation with an ethanol volume fraction up to $20 \%$.

\section{Model simplification for DI water/ethanol mixtures}

The motivation to propose a simplified model is based on the relative comparison of coefficients in equations (5) and (6). It may indeed be emphasized that the influence of $\varepsilon^{\prime}$ on the resonant frequency is approximately 25 times higher than the influence of $\varepsilon$ ". Conversely, the impact of $\varepsilon$ " on the maximum insertion loss is 35 times larger than the influence of $\varepsilon$ '.

In order to fully evaluate the relative values of terms in (5) and (6), values of complex permittivity for DI water/ethanol mixtures are determined by means of a broadband measurement technique [5]. This technique provides reliable values of complex permittivity and stands for our reference in this study. Table II gives the permittivity values of two liquids: pure DI water and a second one with $20 \%$ of ethanol volume fraction $\left(\mathrm{f}_{\mathrm{V}}\right)$ at $20 \mathrm{GHz}$.

TABLE II

COMPLEX PERMITTIVITY OF DI WATER AND DI WATER/ETHANOL MIXTURE $\left(\mathrm{F}_{\mathrm{V}}=20 \%\right)$ AT $20 \mathrm{GHZ}$

\begin{tabular}{ccccc}
\hline \hline Mixture & $\varepsilon^{\prime}(20 \mathrm{GHz})$ & $\varepsilon^{\prime \prime}(20 \mathrm{GHz})$ & $\Delta \varepsilon^{\prime}$ & $\Delta \varepsilon^{\prime \prime}$ \\
\hline DI water & 40.6 & 33.7 & & \\
$\begin{array}{c}\text { DI water/Eth. } \\
(\mathrm{fv}=20 \%)\end{array}$ & 19.7 & 23.2 & 20.9 & 10.5 \\
\hline \hline
\end{tabular}

Table II shows that it is not possible to keep constant either the real part or the imaginary part of the complex permittivity of a DI water/ethanol mixture when the ethanol volume fraction is modified. As a consequence, measured shifts will be due to simultaneous variations of both $\varepsilon$ ' and $\varepsilon$ " parameters. Besides, Table II shows that the permittivity values for both pure DI water and binary liquid mixture (water/ethanol with a volume fraction of ethanol up to $20 \%$ ) are including the range of model validity presented in the last paragraph (see equations (1) to (6)).

As previously outlined, we can assume:

$$
\begin{aligned}
& \frac{<K_{1}>}{<K_{2}>} \approx 25 \\
& \frac{<K_{4}>}{<K_{3}>} \approx-35
\end{aligned}
$$


and, restricting to the investigation of ethanol in aqueous solution with ethanol content up to $20 \%$,

$$
\frac{\Delta \varepsilon^{\prime}}{\Delta \varepsilon^{\prime \prime}} \approx \frac{20.9}{10.5}
$$

It comes:

$$
\left|\frac{\overline{K_{2}} \Delta \varepsilon^{\prime \prime}}{\overline{K_{1}} \Delta \varepsilon^{\prime}}\right| \approx 2.0 \% ;\left|\frac{\overline{K_{3}} \Delta \varepsilon^{\prime}}{\overline{K_{4}} \Delta \varepsilon^{\prime \prime}}\right| \approx 6.4 \%
$$

Equations (10) point out that the contribution of $\varepsilon$ " on the resonant frequency shift and the one of $\varepsilon$ ' on the peak attenuation contrast are negligible. Thus, in the case of DI water/ethanol mixtures - with ethanol volume fraction up to $20 \%$ - the simulation-derived model, which links the resonator's electrical characteristics (stopband frequency and associated attenuation) with the fluid complex permittivity, is reduced to the sensitivity coefficients $\mathrm{K}_{1}$ and $\mathrm{K}_{4}$.

To validate the proposed technique to characterize the complex permittivity of liquid, the measurements of various binary liquid mixture of DI water/ethanol are performed on the fabricated prototype and are presented in the next paragraph.

\section{EXPERIMENTAL RESULTS}

Measurements of the microwave fluid sensor are performed on wafer with a probe station equipped with two $150 \mu \mathrm{m}$ pitch GSG picoprobes connected to an ANRITSU 37369C vector network analyzer (VNA). A thermal chuck system is used to assure a stable liquid temperature of $20^{\circ} \mathrm{C} \pm 0.1^{\circ} \mathrm{C}$. A miniature thermocouple is used to verify the thermal stability of the liquid under investigation. Moreover, we have determined that, under $0 \mathrm{dBm}$ VNA's power level, the temperature increase due to microwave heating is lower than $0.1{ }^{\circ} \mathrm{C}$. Concerning the liquid filling through the structure, the microfluidic channel is connected to a $50 \mu \mathrm{l}$ syringe via a teflon tubing as short as possible to minimize the dead liquid volume. The precise control of the fluidic infusion is realized thanks to a syringe pump from Harvard Apparatus. Flow rate and duration of operation sequences are optimized to efficiently (a few tens of seconds) fill the dead volume and to smoothly fill the microchannel and stabilize the liquid inside (a few tens seconds) before to empty the channel for the next measurement (a few tens of seconds). The probe system is equipped with a microscope allowing the visual inspection of the liquid filling and stabilization inside the channel.

Prior to the measurements, a full-two-port SOLT (Short Open Load Thru) calibration procedure is applied using SUSS MicroTec $|\mathrm{Z}|$ probe CSR 8 calibration standards. The frequency range extends from 16 to $21 \mathrm{GHz}$ and the VNA's power level is fixed to $-10 \mathrm{dBm}$, which avoids any microwave heating. A computerized post-treatment routine is applied in order to extract the resonant frequency and the peak attenuation from the measured S-parameters.

The fabricated stopband resonator is firstly characterized without any injected liquid. Table III summarizes the resonant frequency, loaded and unloaded quality factor and peak attenuation extracted from experiments and from electromagnetic simulations. A good agreement is observed between predicted and measured values, which validate the good operation of the microfabricated structure.

TABLE III

DEVICE CHARACTERISTICS WITHOUT ANY FLUID

\begin{tabular}{ccccc}
\hline \hline & Fr $(\mathrm{GHz})$ & $\begin{array}{c}|\mathrm{S} 21|(@ \mathrm{Fr}) \\
(\mathrm{dB})\end{array}$ & Q0 & QL \\
\hline Simulations & 22.69 & -17.77 & 9.6 & 1.2 \\
Measurements & 22.62 & -16.58 & 8.8 & 1.3 \\
\hline \hline
\end{tabular}

DI water/ethanol mixtures are used as fluids under test, as the complex permittivity of such mixtures is easily changeable by varying the ethanol volume fraction fv [21], [22]. Five different mixtures are measured: pure DI water, mixture M1 with ethanol volume fraction $\mathrm{fv}=1 / 20$, mixture $\mathrm{M} 2$ with $\mathrm{fv}=$ $1 / 10$, mixture $\mathrm{M} 3$ with $\mathrm{fv}=1 / 7$ and mixture $\mathrm{M} 4$ with $\mathrm{fv}=1 / 5$. The five fluids are prepared and injected into the microfluidic channel followed by the measurement of the scattering parameters.

Fig. 5 depicts the modulus of the S21-parameters obtained for the five DI water/ethanol mixtures under measurement. This figure clearly shows that the resonant frequency drifts towards high frequencies, when the ethanol volume fraction increases. It also indicates that the peak attenuation increases, when the ethanol concentration rises.

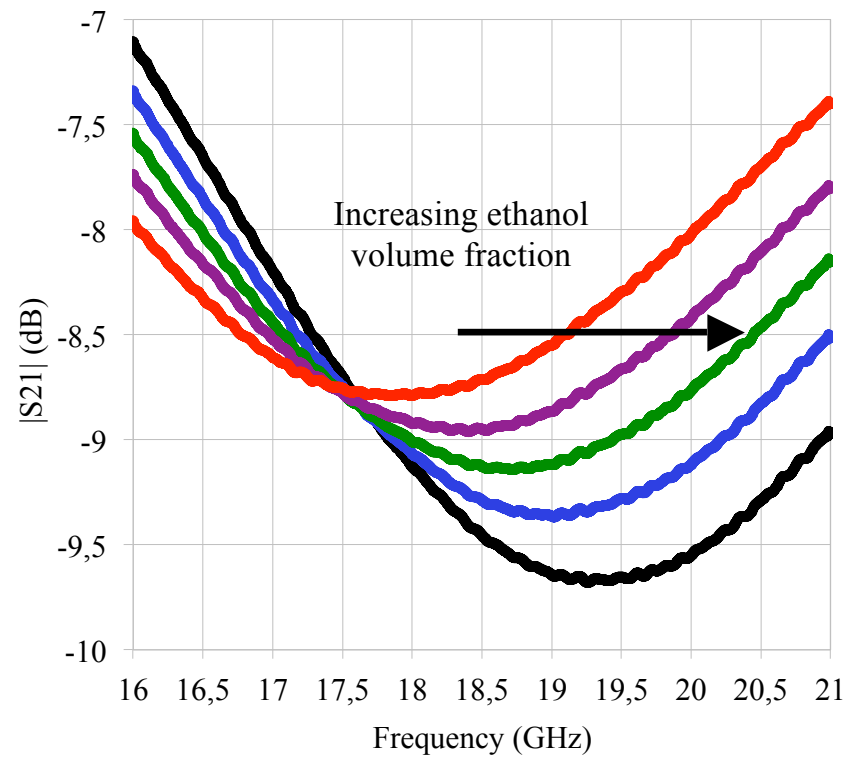

Fig. 5. $|\mathrm{S} 21|(\mathrm{dB})$ for 5 ethanol/DI water mixtures at volume fractions fv : $1 / 5$; $1 / 7 ; 1 / 10 ; 1 / 20 ; 0$ (pure DI water).

Both resonant frequencies and peak attenuation corresponding to the five curves are extracted by means of a computerized post-treatment routine and are listed in Table IV. Moreover, for each mixture, the values of the complex permittivity are determined by means of the already mentioned broadband measurement technique [5] and are listed in the same table. 
TABLE IV

DI WATER/ETHANOL MIXTURES COMPLEX PERMITTIVITY AT THEIR MEASURED RESONANT FREQUENCIES

\begin{tabular}{cccccc}
\hline \hline Mixture & $\mathrm{fv}$ & $\mathrm{Fr}(\mathrm{GHz})$ & $|\mathrm{S} 21|(@ \mathrm{Fr})$ & $\varepsilon^{\prime}$ & $\varepsilon^{\prime \prime}$ \\
\hline DI water & 0 & 17.90 & 0.3638 & 44.7 & 33.8 \\
M1 & 0.05 & 18.39 & 0.3561 & 37.1 & 33.4 \\
M2 & 0.1 & 18.69 & 0.3495 & 30.5 & 29.5 \\
M3 & 0.14 & 18.98 & 0.3409 & 26.0 & 27.4 \\
M4 & 0.2 & 19.34 & 0.3286 & 20.6 & 23.7 \\
\hline \hline
\end{tabular}

Fig. 6.a and Fig. 6.b indicate (red boxes), respectively as functions of the real and imaginary part of the fluid permittivity, the measured electrical characteristics shifts calculated from values in Table IV and defined with:

$$
\begin{gathered}
\Delta F_{r_{M i}}=F_{r_{M i}}-F_{r_{H_{2} O} O} \\
\Delta|S 21|_{M i}=|S 21|_{M i}-|S 21|_{H_{2} O}
\end{gathered}
$$

where $\mathrm{Fr}_{\mathrm{Mi}}, \mathrm{Fr}_{\mathrm{H} 2 \mathrm{O}},|\mathrm{S} 21|_{\mathrm{Mi}}$ and $|\mathrm{S} 21|_{\mathrm{H} 2 \mathrm{O}}$ refer to the measured resonant frequency and rejection of the mixture $\mathrm{Mi}(\mathrm{i}=1$ to 4$)$ and pure DI water respectively.

Similarly, real and imaginary parts of the relative permittivity variations are defined as follows:

$$
\begin{gathered}
\Delta \varepsilon^{\prime}{ }_{M i}=\varepsilon^{\prime}{ }_{M i}-\varepsilon^{\prime}{ }_{H_{2} O} \\
\Delta \varepsilon^{\prime \prime}{ }_{M i}=\varepsilon^{\prime \prime}{ }_{M i}-\varepsilon^{\prime \prime}{ }_{H_{2} O}
\end{gathered}
$$

where $\varepsilon^{6}{ }_{\mathrm{Mi}}$ and $\varepsilon^{\prime \prime}{ }_{\mathrm{Mi}}$ are the real and imaginary parts of the relative permittivity of the mixture $\mathrm{Mi}$ at $\mathrm{Fr}_{\mathrm{Mi}}$ and $\varepsilon^{6}{ }_{\mathrm{H} 2 \mathrm{O}}$ and $\varepsilon^{\prime \prime}{ }_{\mathrm{H} 2 \mathrm{O}}$ correspond to the real and imaginary part of the relative permittivity of DI water at $\mathrm{Fr}_{\mathrm{H} 2 \mathrm{O}}$.

In this study, pure DI water can thus be seen as the reference fluid from which all variations and shifts are derived. Consequently, the origin of the graph depicted in Fig. 6.a and 6.b corresponds to pure DI water.

For comparison purpose, simplified model-based resonant frequency shifts and peak attenuation contrasts are calculated as follows:

$$
\begin{gathered}
\Delta F_{r_{M i}}^{M O D}=K_{1} \times \Delta \varepsilon^{\prime}{ }_{M i} \\
\Delta|S 21|_{M i}^{M O D}=K_{4} \times \Delta \varepsilon_{M i}^{\prime \prime}
\end{gathered}
$$

where $\Delta F_{r_{M i}}^{M O D}$ and $\Delta S 21_{M i}^{M O D}$ are respectively the resonant frequency shifts and peak attenuation contrasts (relative to water) that can be predicted thanks to the model presented in IV.B and IV.C. These two electrical characteristics are presented in Fig. 6.a and Fig. 6.b (solid lines) respectively as functions of the real and imaginary part of the fluid permittivity.

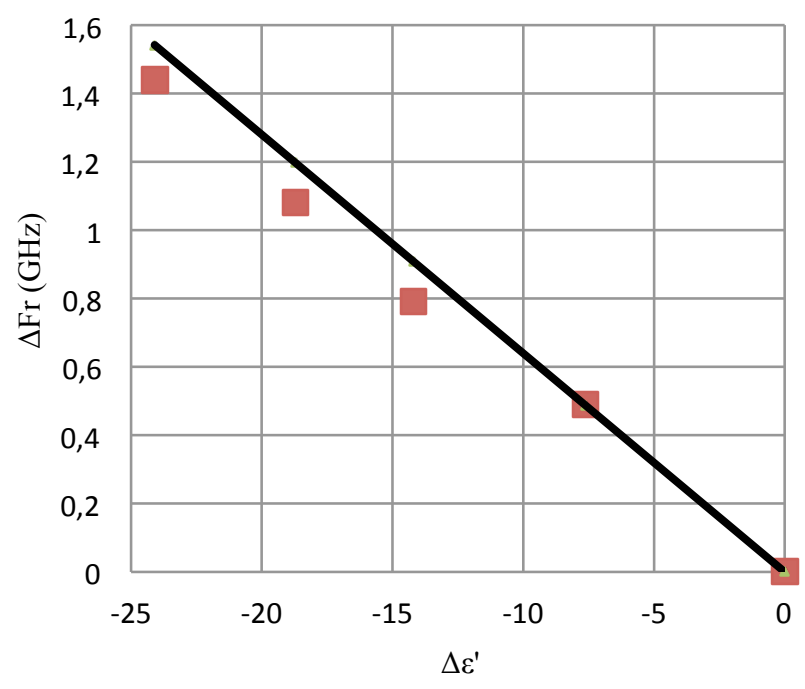

(a)

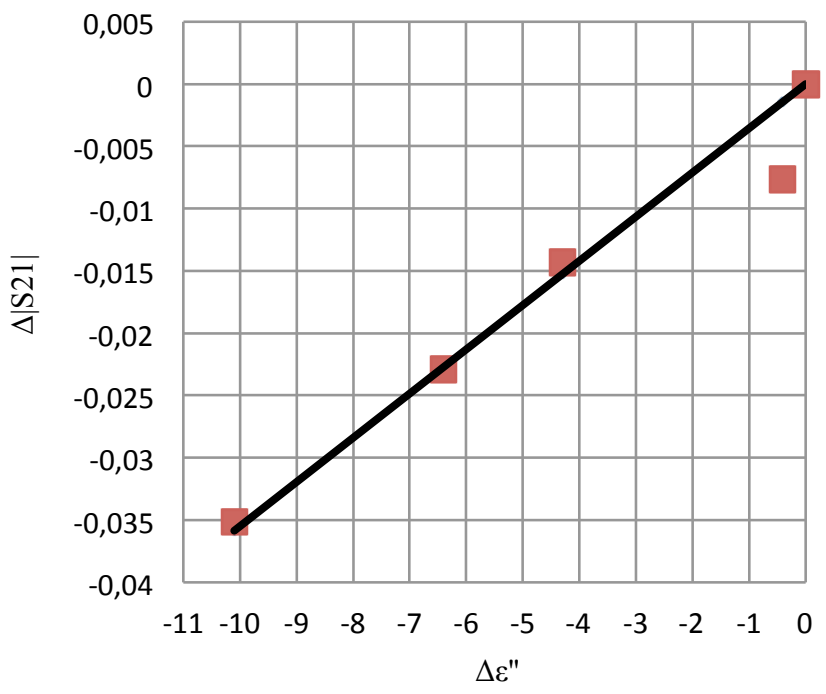

(b)

Fig. 6. (a) Modeled (line) and measured (dots) resonant frequency shifts and (b) modeled (line) and measured (dots) maximum rejection attenuation, due to relative permittivity variations. Origin $(0 ; 0)$ corresponds to DI water.

Fig. 6 demonstrates the good experimental validity of the simplified simulation-derived model at least over the given range of permittivity (which roughly extends from 20 to 44). As an example, resonant frequency shifts obtained for the four mixtures are in good agreement with those predicted by the model. Measured shifts for mixtures M1 to M4 are indeed $0.46 \mathrm{GHz}, 0.86 \mathrm{GHz}, 1.13 \mathrm{GHz}$ and $1.46 \mathrm{GHz}$ respectively, while the proposed model predicts $0.48 \mathrm{GHz}, 0.90 \mathrm{GHz}, 1.18$ $\mathrm{GHz}$ and $1.52 \mathrm{GHz}$ for the same mixtures.

To quantify the discrepancies between the measured and theoretical values, the standard-deviation 'std' are defined as follows : 


$$
\begin{gathered}
\operatorname{std}(\Delta F r)=\sqrt{\frac{1}{4} \sum_{i=1}^{4}\left(\Delta F_{r_{M i}}-\Delta F_{r_{M i}}^{M O D}\right)^{2}} \\
\operatorname{std}(\Delta|S 21|)=\sqrt{\frac{1}{4} \sum_{i=1}^{4}\left(\Delta|S 21|_{M i}-\Delta|S 21|_{M i}^{M O D}\right)^{2}}
\end{gathered}
$$

where $\Delta \mathrm{Fr}_{\mathrm{Mi}}$ and $\Delta F_{r_{M i}}^{M O D}$ refer to (11) and (14) respectively and $\Delta|\mathrm{S} 21|_{\mathrm{Mi}}$ and $\Delta|S 21|_{M i}^{M O D}$ refer to (12) and (15) respectively.

A numerical estimation of the standard deviation $\operatorname{std}(\mathrm{Fr})$ gives $\operatorname{std}(\mathrm{Fr})=0.083 \mathrm{GHz}$. The relative uncertainty $\delta \mathrm{Fr} / \mathrm{Fr}$ (in case of mixture M1 - worst case) is thus :

$$
\frac{\delta F r}{F r}=\frac{s t d(F r)}{F r}=\frac{0.083}{18.4}=0.5 \%
$$

and the relative uncertainty on the real part of the relative permittivity (in case of mixture M4 - worst case) is :

$$
\frac{\delta \varepsilon^{\prime}}{\varepsilon^{\prime}}=\frac{\left.s t d(F r) /<K_{1}\right\rangle}{\varepsilon^{\prime}}=\frac{0.083 / 0.0631}{20.6}=6.4 \%
$$

The same analysis is performed on the peak attenuation and the imaginary part of the relative fluid's permittivity. The standard deviation of the peak attenuation is estimated at $\operatorname{std}(\Delta|\mathrm{S} 21|)=0.0032 \mathrm{~dB}$, which translates, in the worst case, to an accuracy of the predicted model of $\delta|\mathrm{S} 21 / /| \mathrm{S} 21 \mid$ of around 1 $\%$ and then to a maximum relative uncertainty on the imaginary part of the relative permittivity of :

$$
\frac{\delta \varepsilon^{\prime \prime}}{\varepsilon \prime \prime}=\frac{\left.s t d(\Delta|S 21|) /<K_{4}\right\rangle}{\varepsilon^{\prime \prime}}=\frac{0.0032 / 0.00356}{23.7}=3.8 \%
$$

As already mentioned, pure DI water sets the reference fluid from which all contrasts are derived. The reported accuracies consequently correspond to parameter (permittivity for instance) contrasts with respect to DI water and not to the absolute values of these parameters.

The reached accuracy values combined to the results presented in this paragraph illustrate the concept of an accurate and efficient resonant-based device for complex permittivity characterization of aqueous solutions. Other techniques available in the literature enable a permittivity characterization with relative uncertainty of few percent [12], [23], [24]. The presented results show that accurate liquid's permittivity characterization can be achieved with a fully integrable resonant-based sensor operating in K-band.

\section{CONCLUSION}

The microwave and microfluidic sensor presented in this paper meets the specifications to develop an integrated platform workable to perform efficient and accurate complex permittivity characterization of aqueous solutions. The compactness of the device is due to the convergence between microtechnology for microwave circuits and microfluidic, which is, suited to a lab-on-a-chip approach. Microfluidic structures make it possible to carry out measurements on nanolitter range fluids in a predictable and repeatable manner.
As far as the efficiency of the methodology is concerned, a resonant-based device is chosen as the complex permittivity characterization simply relies on the measurement of the resonant frequency and associated attenuation. A simple and linear model for resonant frequency and peak attenuation shifts due to complex permittivity variations is derived from simulations. The measurements of the fabricated prototype with DI water/ethanol mixtures confirm the validity of the model over a range of relative permittivity, which extends from 20 to 40. A good accuracy is reached, as the relative uncertainty on the permittivity never exceeds $6.5 \%$ over the above-cited range. As a result, the presented microwave and microfluidic sensor opens a way towards the design and fabrication of a fully integrated platform dedicated to bio or chemical sensing.

\section{REFERENCES}

[1] H. P. Schwan, "Electrical properties of tissues and cell suspensions: Mechanism and models," in Proc. 16th Annu. IEEE Int. Conf., 1994, vol. 1, pp. A70-A71.

[2] M. A. Stuchly, A. Kraszewski, S. S. Stuchly, and A. M. Smith, "Dielectric properties of animal tissues in vivo at radio and microwave frequencies: Comparison between species," Phys. Med. Biol., vol. 27, no.7, pp. 927-936, 1982.

[3] Z. Zhu and A. S. Waggoner, "Molecular mechanism controlling the incorporation of fluorescent nucleotides into DNA by PCR", Cytometry, 28:206-211, January, 2007.

[4] M. L. Larramendy, Wa'el El-Rifai and S. Knuutila, "Comparison of fluorescin isothiocyanate- and Texas red-conjugated nucleotides for direct labeling in comparative genomic hybridization", Cytometry, 31:174-179, October 1997.

[5] K. Grenier, D. Dubuc, P. E. Poleni, M. Kumemura, H. Toshiyoshi, T. Fujii and H. Fujita, "Integrated broadband microwave and microfluidic sensor dedicated to bioengineering", IEEE Transactions on Microwave Theory an Techniques, vol. 57, no. 12, pp. 3246-3253, December 2009.

[6] F. Kremer and A. Schönhals, Broadband Dielectric Spectroscopy, Springer-Verlag, Berlin, 2003.

[7] S. Jenkins, T. E. Hodgets, R. N. Clarke and A. W. Preece, "Dielectric measurements on reference liquids using automatic network analysers and calculable geometries", Measurement Science and Technology 1, pp. 691-702, February 1990.

[8] J. M. Català-Civera, A. J. Canós, F. L. Peňaranda-Foix and E. de los Reyes Davó, "Accurate determination of the complex permittivity of materials with transmission reflection measurement in partially filled rectangular waveguides", IEEE Transactions on Microwave Theory and Techniques, vol. 51, no. 1, pp. 16-23, January 2003.

[9] A. P. Gregory and R. N. Clarke, "A review of RF and microwave techniques for dielectric measurements on polar liquids", IEEE Transactions on Dielectrics and Electrical Insulation, vol. 13, no. 4, pp. 727-743, August 2006.

[10] J. Kim, A. Babajanyan, A. Hovsepyan, K. Lee and B. Friedman, "Microwave dielectric resonator biosensor for aqueous glucose solution", Review of Scientific Instruments 79, August 2008.

[11] M. Neshat, S. Gigoyan, D. Saeedkia and S. Safavi-Naeini, "Travelingwave whispering gallery resonance sensor in millimeter-wave range", Electronic Letters, vol. 44, no. 17, August 2008.

[12] H. Kawabata and Y. Kobayashi, "Accurate measurements of complex permittivity of liquid based on a $\mathrm{TM}_{010}$ mode cylindrical cavity method", European Microwave Conference, 2005.

[13] B. Kapilevich and B. Litvak, "Optimized microwave sensor for online concentration measurements of binary liquid mixtures", IEEE Sensors Journal, vol. 11, no. 10, October 2011, pp.2611-2616.

[14] Kapilevich B. Yu, S. G. Ogourtsov, V. G. Belenky, A. B. Maslenikov, and A. S. Omar, "Accurate microwave resonant method for complex permittivity measurements of liquids," IEEE Trans. Microwave Theory Tech., Vol. 48, No. 11, 2159-2164, 2000.

[15] H. J. Lee, H. S. Lee, K. H. Yoo and J. G. Yook, "DNA sensing using split-ring resonator alone at microwave regime", Journal of Applied Physics 108, July 2010. 
[16] Y. H. Kim, K. Jang, Y. J. Yoon and Y. J. Kim, "A novel relative humidity sensor based on microwave resonators and a customized polymeric film", Sensors and Actuators B 117, pp. 315-3222, December 2005.

[17] C. Liu and Y. Pu, "A microstrip resonator with slotted ground plane for complex permittivity measurements of liquids", IEEE Microwave and Wireless Components Letters, vol. 18, no. 4, pp. 257-259, April 2008.

[18] K. Hettak, N. Dib, A. F. Shetta and S. Toutain, "A class of novel uniplanar series resonators and their implementation in original applications", IEEE Transactions on Microwave Theory and Techniques, vol. 46, no. 9, pp. 1270-1276, September 1998.

[19] http://www.ansoft.com/products/hf/hfss/

[20] W. J. Ellison, "Permittivity of pure water, at standard atmospheric pressure, over the frequency range $0-25 \mathrm{THz}$, and the temperature range 0-100 ${ }^{\circ} \mathrm{C}$, Journal of Physical and Chemical Reference Data, vol. 36, no. 1, pp. 1-18, February 2007.

[21] J. Z. Bao, M. L. Swicord and C; C. Davis, "Microwave dielectric characterization of binary mixtures of water, methanol and ethanol', Journal of Chemical Physics, 104 (12), pp. 4441-4450, March 1996.

[22] Y. Wang and M. N. Afsar, "Measurement of complex permittivity of liquids using waveguide techniques", Progress in Electromagnetic Research (PIER) 42, pp. 131-142, 2003.

[23] M. Mosalanejad, G. Moradi and A. Abdipour, "An inverted microstrip resonator for complex permittivity measurement of medium loss liquids using 3D-FDTD simulation", Mediterranean Microwave Symposium, pp. 407-410, August 2010.

[24] X. Yang and K. Huang, "The empirical formula for calculating the complex effective permittivity of an aqueous electrolyte solution at microwave frequency", IEEE Transactions on Geoscience and Remote Sensing, vol. 43, pp. 315-320, February 2005.

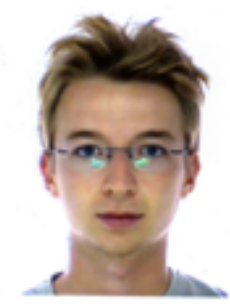

Thomas Chretiennot graduated from the Ecole Nationale Superieure de l'Electronique et de ses Applications (E.N.S.E.A.), Cergy, France, in 2009, a French graduate school in electrical engineering, computer science and telecommunications. He is currently working towards his $\mathrm{PhD}$ degree at the Laboratory of Analyse and Architecture of System part of National Scientific Research Center (LAASCNRS), Toulouse, France. His work deals with the development of microwave and microfluidic

biosensors.

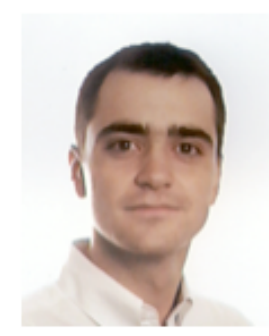

David Dubuc received the Agregation degree from the Ecole Normale Supérieure de Cachan, Paris, France, in 1996, and the M.S. and Ph.D. degrees in electrical engineering from the University of Toulouse, Toulouse, France, in 1997 and 2001, respectively.|par

Since 2002, he has been an Associate Professor with the University of Toulouse, and a Researcher with the Laboratory of Analysis and Architecture of System part of National Scientific Research Center (LAAS-CNRS), Toulouse, France. From 2007 to 2009, he was a Visiting Senior Researcher with the Laboratory of Integrated Micromechatronic Systems (LIMMS-CNRS)/Institute of Industrial Science (IIS), University of Tokyo, Tokyo, Japan. His research interests include the development of microwave circuits integrated due to microtechnologies and their application to wireless telecommunication and biology.

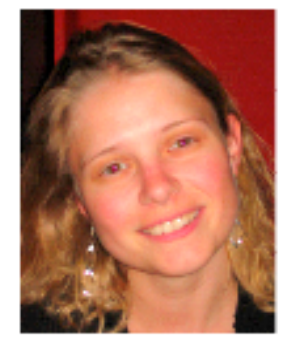

Katia Grenier (M'03) received her M.S. and Ph.D. degrees in electrical engineering from the University of Toulouse, Toulouse, France, in 1997 and 2000, respectively. She was engaged in microelectromechanical systems (MEMS) circuits on silicon. She was a Postdoctoral Fellow at Agere Systems (Bell Labs). In 2001, she joined the Laboratory of Analysis and Architecture of System of the National Scientific Research Center (LAAS-CNRS), Toulouse, France. From 2007 to 2009, she was with the Laboratory for Integrated Micromechatronic Systems CNRS (LIMMS-CNRS)/Institute of Industrial
Science (IIS), Universtity of Tokyo, Tokyo, Japan, where she was engaged in launching research activities on microwave-based biosensors. Her research interests in LAAS-CNRS are now focused on the development of fluidicbased micro/nanosystems for biological and medical applications as well as for reconfigurable wireless ones. Dr. Grenier is a member of the IEEE MTT10 Technical Committee on Biological effect and medical applications of RF and microwave of the IEEE Microwave Theory and Techniques Society. 